\title{
CD31 and D2-40 Contribute to Peritoneal Metastasis of Colorectal Cancer by Promoting Epithelial-Mesenchymal Transition
}

\author{
Xinqiang Zhu' ${ }^{1}$ Gang Zhou ${ }^{2}$, Peng Ni ${ }^{3}$, Xuetong Jiang ${ }^{1}$, Hailong Huang ${ }^{1}$, Jianqiang $\mathrm{Wu}^{1}$, Xiaohong Shi ${ }^{4}$, \\ Xiaoling Jiang ${ }^{4}$, and Jianing Liu ${ }^{5}$ \\ ${ }^{1}$ Department of General Surgery, The Affiliated Suqian Hospital of Xuzhou Medical University, Suqian, ${ }^{2}$ Department of Gastrointestinal \\ Pancreatic Surgery, The Affiliated Jiangning Hospital of Nanjing Medical University, Nanjing, ${ }^{3}$ Department of General Surgery, Suining \\ County Hospital of Traditional Chinese Medicine, Suining, and Departments of ${ }^{4}$ Pathology and ${ }^{5}$ Digestion, The Affiliated Suqian \\ Hospital of Xuzhou Medical University, Suqian, China
}

\section{Article Info}

Received December 2, 2019

Revised March 12, 2020

Accepted March 25, 2020

Published online May 13, 2020

\section{Corresponding Author \\ Jianing Liu \\ ORCID https://orcid.org/0000-0002-3373-4241 \\ E-mail Ljn2019@sina.com}

Xinqiang Zhu and Gang Zhou contributed equally to this work as first authors.
Background/Aims: Colorectal cancer (CRC) patients often exhibit peritoneal metastasis, which negatively impacts their prognosis. CD31 and D2-40 have recently been suggested to be predictors of breast cancer prognosis, but their role in colorectal peritoneal metastasis (CRPM) remains unknown.

Methods: The expression profiles of CD31 and D2-40 were analyzed in CRC patients with or without CRPM and in CRC cell lines with increasing metastatic potential. Overexpression and short hairpin RNA knockdown assays were performed in CRC cells, and the effects of these alterations on epithelial-mesenchymal transition (EMT) in vitro, growth of xenograft tumors in vivo, and peritoneal metastasis potential in a mouse model of CRPM were examined.

Results: The expressions of CD31 and D2-40 were upregulated in CRC tumor tissues and was elevated further in tumor tissues from patients with CRPM. CD31 and D2-40 expression levels exhibited increasing trends parallel to the EMT potential of CRC cells. CD31 and D2-40 are essential for CRC cell EMT in vitro as well as for xenograft tumor growth and peritoneal metastasis in vivo.

Conclusions: CD31 and D2-40 contribute to CRPM by promoting EMT and may serve as prognostic markers and therapeutic targets for CRC, particularly in patients with peritoneal metastasis. (Gut Liver 2021;15:273-283)

Key Words: Colorectal neoplasms; Peritoneal metastasis; CD31; D2-40; Epithelial-mesenchymal transition

\section{INTRODUCTION}

Colorectal cancer (CRC) is listed after lung, esophageal and breast cancer as the fourth most common malignancy in humans around the world, with more than 1.2 million new incidences being diagnosed in 2008 alone according to the cancer registry data. ${ }^{1}$ The etiology of CRC is closely associated with diet, old age, smoking, obesity or inadequate physical exercises, and inherited genetic disorders are also responsible for a small portion of morbidity. ${ }^{2}$ Screening is often recommended to the elderly with preventive purposes, and diagnosis is usually established based on biopsy during colonoscopy and sigmoidoscopy. ${ }^{3}$ The main options for clinical intervention of CRC include chemotherapy, radiotherapy, combinations of surgery, and targeted therapies. ${ }^{4}$ Over the past decades with advance in the crucial understanding of CRC, particularly in regard to histological features, early diagnosis, as well as staging, the survival has been notably improved. ${ }^{5}$ However, for patients in the advance stage, the efficacy and applicability of therapies remain rather limited. Therefore, more comprehensive insights into this disease are still urgently needed.

Up to one-fifth of the patients with CRC exhibit synchronous distant dissemination at diagnosis, ${ }^{6} 4 \%$ of whom 
have isolated peritoneal spread. ${ }^{7}$ In addition to the lymphatic ad hematogenous dissemination, colorectal tumor cells can directly spread to the peritoneum through the transcoelomic route, resulting in colorectal peritoneal metastasis (CRPM). CRPM occurs either metachronously or synchronously to the primary tumor in $8 \%$ to $20 \%$ of CRC patients. ${ }^{8}$ A prior investigation analyzed 11,124 CRC patients and found diagnosis of CRPM in $8.3 \%$ of patients. ${ }^{7}$ Another recent analysis of 5,671 patients, who underwent operation for CRC and were followed up at least 5 years, ${ }^{9}$ reported that metastases were developed in 1,042 patients (18\%), and were located in the peritoneum in 197 patients (19\%), in up to $40 \%$ of which the peritoneum was the unique metastatic site.

Lymphatic and blood vessel invasion to local or regional lymph nodes, as well as distant sites, occurs in the initial stages of tumor metastasis. Lymphovascular invasion is suggested as a predictor of postoperative prognosis by the St. Gallen consensus guidelines. ${ }^{10}$ However, lymphovascular invasion is often revealed with the use of hematoxylin and eosin stained material, which does not distinguish blood vessel invasion from lymphatic invasion. ${ }^{11}$ Although there are methodological challenges to distinguish lymphatic from blood vessels, a number of specific markers have been described, including blood vessel invasion marker CD31 and lymphatic invasion marker D2-40, ${ }^{12}$ which have recently been suggested as a predictor of breast cancer prognosis. ${ }^{13} \mathrm{CD} 31$ is a $130-\mathrm{kDa}$ transmembrane glycoprotein platelet endothelial cell adhesion molecule-1, which belongs to the immunoglobulin superfamily. ${ }^{12} \mathrm{D} 2-$ 40 is an IgG2a monoclonal antibody to the oncofetal M2A antigen, and is often expressed in fetal testis and germ cell tumors. ${ }^{14}$ To date, there has been no investigation conducted on the role of either CD31 or D2-40 in CRPM of CRC patients.

In the current study, we analyzed the expression profiles of CD31 and D2-40 among CRC patients with or without CRPM, and further explored their role and underlying molecular mechanism in CRPM using a mouse model.

\section{MATERIALS AND METHODS}

\section{Patients and tissue samples}

CRC tumor and adjacent normal tissue samples were collected from 80 CRC patients treated in the Affiliated Suqian Hospital of Xuzhou Medical University between January 2015 and October 2018. All patients provided written informed consent prior to the sample collection, and the study obtained approval from the Institutional Review Board of the Affiliated Suqian Hospital of Xuzhou
Medical University (IRB number: SQYY2014032).

\section{Immunohistochemistry}

All tissue samples were subjected to fixation in $10 \%$ neutral buffered formalin and paraffin embedment with standard surgical pathology protocols. Diagnoses were performed on hematoxylin and eosin-stained slides with standard histopathologic criteria. Immunohistochemistry was carried out on one single representative block from each sample. Tissue sections at $5 \mathrm{~mm}$ thickness were dewaxed and then incubated at $120^{\circ} \mathrm{C}$ for 5 minutes in citrate buffer (pH 6) in an electric pressure cooker for antigen retrieval. Next, sections were treated in $3 \%$ hydrogen peroxide for 5 minutes to quench endogenous peroxidase, followed by incubation with primary monoclonal antibodies specific for CD31 (1:80, DAKO Corp., Carpinteria, CA, USA) or D2-40 antigen (1:5, Signet Laboratories, Dedham, MA, USA) for 25 minutes at ambient temperature. After washes to remove unbound primary antibodies, sections were incubated with commercial biotinylated secondary antiimmunoglobulin, followed by incubation with avidin coupled to biotinylated horseradish peroxidase, both at room temperature (LSAB2 kit, DAKO Corp.). Diaminobenzidine was used as the chromogenic peroxidase substrate. Lastly, sections were counterstained using hematoxylin after immunohistochemical reactions. Negative controls, in which primary antibody was omitted, and positive controls of appropriate cytoplasmic reaction for each antigen were included to verify specificity.

\section{Microvessel density assessment}

Assessment of microvessel density using immunohistochemical assays against CD31 or D2-40 antigen was conducted following the Weidner method as previously described. ${ }^{15}$ Evaluations were conducted by two investigators blind to patient grouping, with a microscope with two heads. Two "hot-spots," regions that were most vascularized within a tumor sample, were selected at low magnification $(\times 40)$. For each region, vessels in one representative field at high magnification $\left(\times 400 ; 0.152 \mathrm{~mm}^{2} ; 0.44 \mathrm{~mm}\right.$ diameter) were counted. The representative high magnification fields were labeled for future image cytometric analyses. Isolated endothelial cells that were immunoreactive, as well as clusters of endothelial cells separate from other microvessels, were identified as individual microvessels. Nonspecific labeling of nonendothelial structures, or endothelial staining of large vessels containing tunica media, were excluded. Mean visual density of microvessels for $\mathrm{CD} 31$ or D2-40 was quantified using the average value of four counts (two microscopic fields by each of the two investigators). 


\section{Cell lines}

SW480, SW620 and SW620s5 (S5) CRC cell lines were obtained from and authenticated by the American Type Culture Collection (Rockville, MD, USA). All cells were cultured using PRMI-1640 medium with supplementation of $10 \%$ fetal bovine serum (HyClone; Logan, UT, USA) and $1 \%$ penicillin-streptomycin-glutamine (Gibco; Grand Island, NY, USA). The cell cultures were maintained in 5\% $\mathrm{CO}_{2}$ incubator at $37^{\circ} \mathrm{C}$.

\section{Real-time polymerase chain reaction}

The relative messenger RNA expression was assessed by quantitative polymerase chain reaction (PCR). In brief, total RNA was extracted from cell samples using Trizol Reagent (Invitrogen, Carlsbad, CA, USA). The quantity, as well as quality of RNA samples, were verified using Bioanalyzer 2100 (Agilent, Santa Clara, CA, USA). One microgram total RNA from each sample was subjected to reverse transcription with the SuperScript III First-Strand Synthesis SuperMix (ThermoFisher, Waltham, MA, USA). The real-time polymerase chain reaction (RT-PCR) was conducted using the PowerUp SYBR ${ }^{\mathrm{TM}}$ Green Master Mix (ThermoFisher) according to the provided protocol. The expression levels relative to GAPDH were determined using the $2^{-\Delta \Delta \mathrm{Ct}}$ method. The primers used were as follows: E-cadherin forward (5'-TCG AGG GAA AAA TAG GCT G-3'), E-cadherin reverse (5'-CCG AGA GCT ACA CGT TCA C-3'), N-cadherin forward (5'-GCA TAC ACC ATG CCA TCT T-3'), N-cadherin reverse (5'-TGC ATG AAG GAC AGC CTC T -3'), CD31 forward (5'-AAC AGT GTT GAC ATG AAG AGC C-3'), CD31 reverse (5'-TGT AAA ACA GCA CGT CAT CCT T-3'), D2-40 forward (5'-CCA GCG AAG ACC GCT ATA AGT C-3'), D2-40 reverse (5'CTC GAT GCG AAT GCC TGT TA-3'), GAPDH forward (5'-GGA GCG AGA TCC CTC CAA AAT-3'), GAPDH reverse (5'-GGC TGT TGT CAT ACT TCT CAT GG-3').

\section{Western blot}

Cell lysates were prepared in ice-cold radioimmunoprecipitation buffer. Protein samples of $15 \mu \mathrm{g}$ each were separated through sodium dodecyl sulfate-polyacrylamide gel electrophoresis and transferred onto polyvinylidene fluoride (PVDF) membranes (Roche, MO, USA), which were then incubated with primary antibodies against E-cadherin (\#3195, 1:1,000), N-cadherin (\#13116, 1:1,000) and GAPDH (\#2118, 1:1,000), purchased from Cell Signaling Technology (Danvers, MA, USA), overnight at $4^{\circ} \mathrm{C}$. After being thoroughly washed with TBST, the membranes were incubated for 1 hour with horseradish peroxidase-conjugated secondary antibody (\#7074, 1:5,000), purchased from Cell Signaling Technology (Danvers). Visualization of the final blots was achieved with the use of ECL kit (Millipore, St. Charles, MO, USA).

\section{Cell detachment and attachment assay}

The cells were seeded in triplicate at the density of $4 \times 10^{4}$ cells per well into 24 -well plates. After 24 hours incubation at $37^{\circ} \mathrm{C}$, unattached cells were rinsed off, whereas attached cells were treated at $37^{\circ} \mathrm{C}$ for 3 minutes using $0.05 \%$ trypsin. Next, fresh medium was added to terminate the activity of trypsin, and detached cells were harvested for counting. The remaining cells were subjected to another incubation with $0.25 \%$ trypsin to detach and then be counted. Data were presented as number of detached cells/(number of detached cells+number of remaining cells) $\times 100 \%$, and as number of attached cells/numbers of total cells $\times 100 \%$.

\section{Overexpression and knockdown assays}

Overexpression and short hairpin RNA silencing of CD31 and D2-40 were performed using lentiviral transduction. The cells during the exponential growth were seeded into 6-well plates and transfected with lentivirus containing CD31 and D2-40 cDNA/short hairpin RNA. After 24 hours, the cells were transferred to $10 \mathrm{~cm}$ petri dish for puromycin and/or neomycin resistance selection. The efficiency of overexpression as well as knockdown $\mathrm{KD})$ was verified through real-time polymerase chain reaction.

\section{Experimental mice}

The BALB/cByJ mice were obtained from Vitalstar, Beijing, China, and housed in vivarium maintained under constant temperature of $21^{\circ} \mathrm{C}$, relative humidity of $50 \%$ to $60 \%$, and photoperiod (reversed 12-hour light/12-hour dark cycle with lights on between 7:00 PM and 7:00 AM). All animal experimental procedures were conducted in compliance with protocols approved by the Animal Care and Use Committee of the Affiliated Suqian Hospital of Xuzhou Medical University (approval number: SQYYJCYJ201).

\section{CRC xenograft mouse model}

The 4 to 6 weeks old BALB/cByJ mice were randomly assigned into each group, with six mice per group. The $10^{6}$ CRC cells were prepared as $100 \mu \mathrm{L}$ single cell suspension, and inoculated subcutaneously into both lower flanks for establishment of xenograft tumors. Tumor diameters were measured using digital calipers on days indicated for up to 15 days, and the volumes of the tumors were calculated using the formula: xenograft size $(\mathrm{mL})=(\text { width })^{2} \times$ length $/ 2$. During the 15-day study period, mice did not exhibit any 
indications of suffering or obvious alterations in social behaviors, nor did we observe any physiological complications on the animals. On day 15, all mice were euthanized via $\mathrm{CO}_{2}$ inhalation, followed by extraction of the xenograft to measure its weight on a digital balancer accurate to $0.001 \mathrm{~g}$.

\section{CRPM mouse model}

CRPM in mouse model was established according to previous methods. ${ }^{16}$ The 8 to 12 weeks old BALB/cByJ mice were randomly assigned into each group, with eight mice per group. Briefly, the mouse was anesthetized through an intraperitoneal injection of ketamine/xylazine mixture and the skin over the abdomen was disinfected. A midline laparotomy incision about $1 \mathrm{~cm}$ long was generated. The parietal peritoneum was repeatedly scraped with a cotton swab while the peritoneum was held gently using forceps. The peritoneal cavity was subsequently closed in planes by a single running suture with $4-0$ Vicril. The skin was also sutured back using a simple running suture with 5-0 Prolene. CRC cells were then delivered into the cavity via injections into the left hypochondrium quadrant of the ab- domen through a 12-gauge needle. All animals recovered after the surgeries without infections or complications, and were closely monitored after the study started. During the 15-day study period, the animals did not exhibit any physiological complication, or indications of suffering or altered social behaviors.

\section{Peritoneal carcinomatosis index}

Fifteen days after grafting of cells, all animals were euthanized via $\mathrm{CO}_{2}$ inhalation. Complete midline laparotomy was immediately performed, and the abdominal cavity was separated into 13 zones for inspection. The peritoneal carcinomatosis index (PCI) scores were determined as follows: 0 indicates no macroscopic tumor; 1 indicates limited tumor growth (diameter of 1-2 mm); 2 indicates moderate tumor growth (diameter of $2-4 \mathrm{~mm}$ ); and 3 indicates abundant tumor nodules (diameter $>4 \mathrm{~mm}$ or deposits $>5)^{17}$
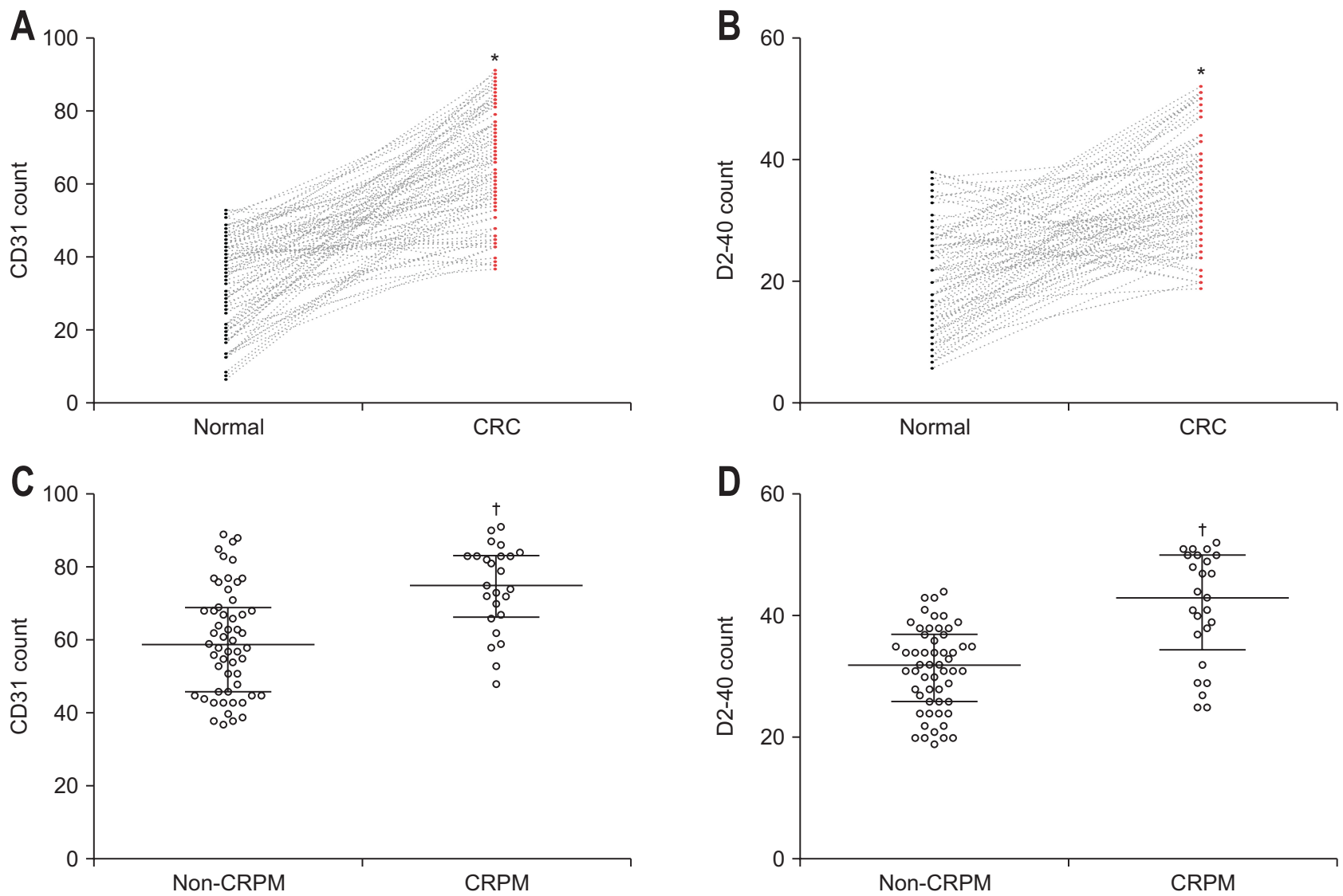

Fig. 1. Assessment of microvessel density assessment by CD31 and D2-40 levels in colorectal cancer (CRC) patient tissues. (A) CD31 and (B) D2-40 levels were compared between tumor and adjacent normal tissue samples from patient with CRC ( $n=80$ pairs). (C) CD31 and (D) D2-40 levels were compared between tumor tissues from non-colorectal peritoneal metastasis (CRPM) patients ( $n=55$ ) and CRPM patients $(n=25) .{ }^{*} p<0.0001$, paired comparison between two groups; ${ }^{\dagger} p<0.0001$, unpaired comparison between two groups. 


\section{Statistical analysis}

All data presented in the current study were obtained from at least three independent experiments. The data analysis was conducted using SPSS 23.0 software (IBM Corp., Armonk, NY, USA), and one-way analysis of variance followed by Tukey multiple comparison test was used for statistical comparisons. $\mathrm{p}$-values less than 0.05 were considered as statistically significant.

\section{RESULTS}

\section{Microvessel density in CRC patient tissues}

Eighty CRC patients were recruited in the current study, and their CRC tumor tissues and paired adjacent normal tissues were collected for microvessel density analysis, us- ing either CD31 or D2-40 immunohistochemistry staining counts. ${ }^{15}$ As shown in Fig. 1A and B, compared to their paired normal tissues, counts of CD31 and D2-40 were both significantly elevated in CRC tumor tissues ( $\mathrm{p}<0.0001$ paired comparison).

Among the 80 CRC patients, 25 were diagnosed with CRPM. We therefore further divided them into nonCRPM group $(n=55)$ and CRPM group $(n=25)$, and compared their CD31 and D2-40 counts in this setting. To our surprise, counts of both CD31 (Fig. 1C) and D2-40 (Fig. $1 D)$ were higher in the CRPM group than the non-CRPM group, with statistical significance $(\mathrm{p}<0.0001$ unpaired comparison). These data indicated that elevated expression of CD31 and D2-40 might be linked with peritoneal metastasis of CRC.
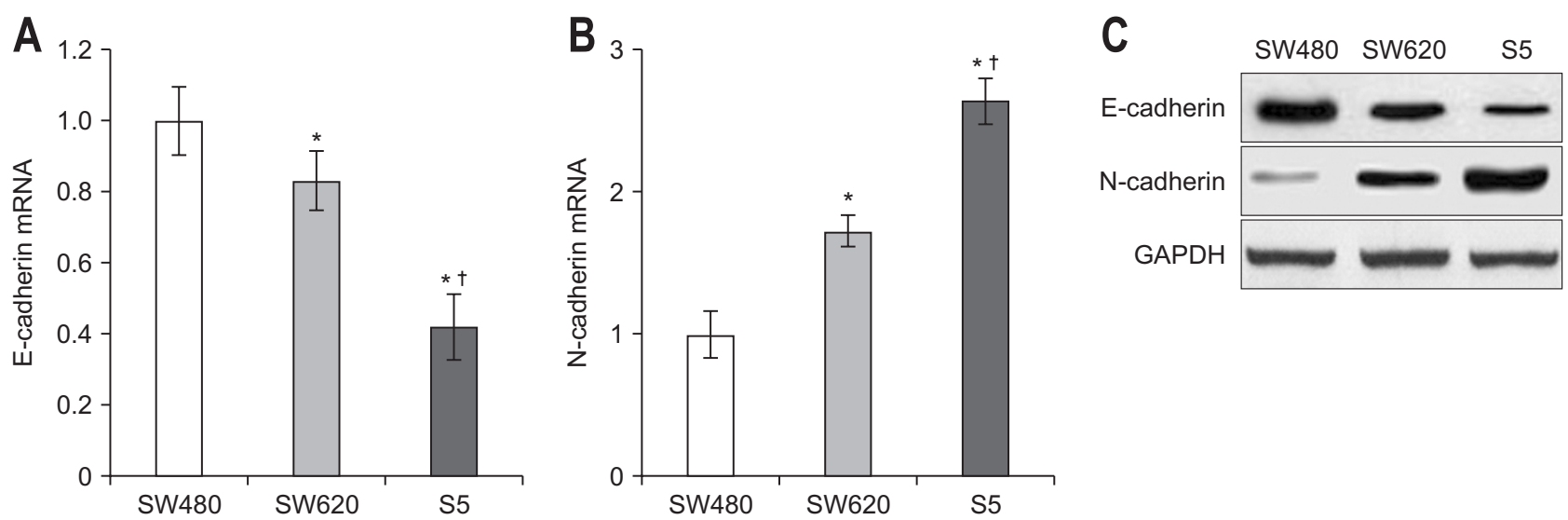

Fig. 2. Three colorectal cancer (CRC) cell lines with increasing epithelial-mesenchymal transition potential. (A) E-cadherin and (B) N-cadherin messenger RNA (mRNA) expression levels were assessed by real-time polymerase chain reaction in the SW480, SW620 and SW620s5 (S5) CRC cell lines. Values are the mean $\pm S D$ of at least three biological replicates. (C) E-cadherin and $\mathrm{N}$-cadherin protein expression levels in the SW480, SW620 and S5 CRC cell lines were assessed by Western blotting. ${ }^{*} p<0.05$, comparison between SW480 cells and either SW620 or S5 cells; ${ }^{\dagger} \mathrm{p}<0.05$, comparison between SW620 with S5.
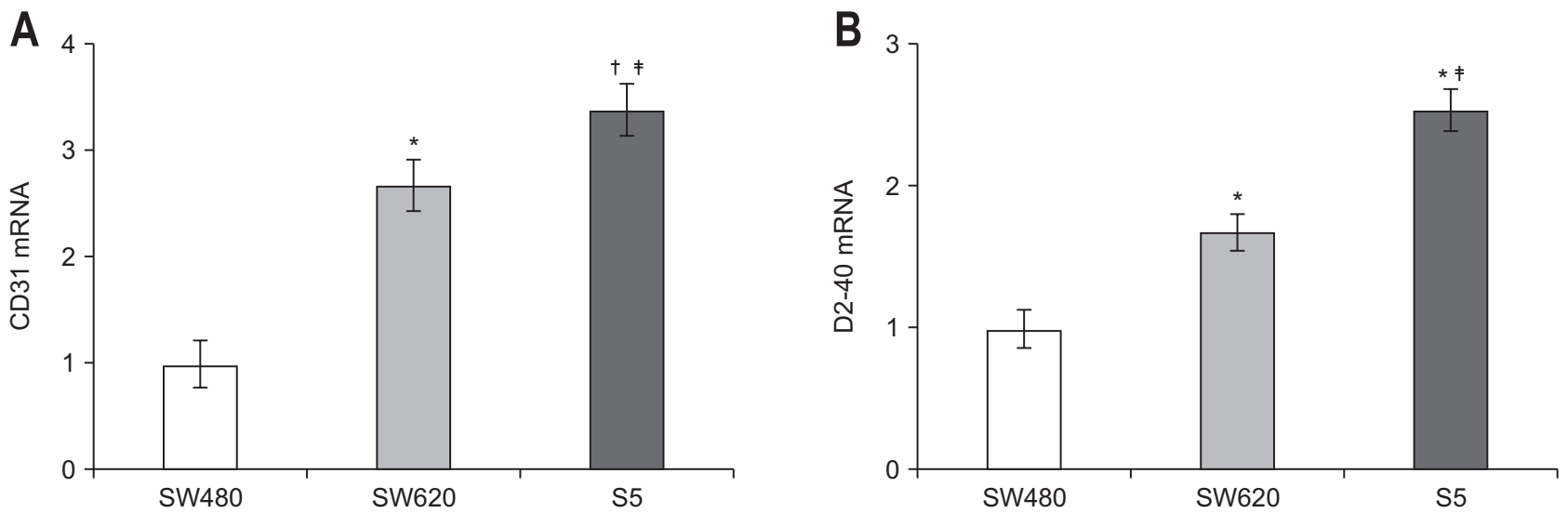

Fig. 3. CD31 and D2-40 expression levels exhibit increasing trends in parallel to the epithelial-mesenchymal transition potential of colorectal cancer (CRC) cell lines. (A) CD31 and (B) D2-40 messenger RNA (mRNA) expression levels were assessed by real-time polymerase chain reaction in the SW480, SW620 and SW620s5 (S5) CRC cell lines. Values are the mean \pm SD of at least three biological replicates. ${ }^{*} p<0.05$, ${ }^{\dagger} p<0.01$, comparison between SW480 cells and either SW620 or S5 cells; ${ }^{\ddagger} p<0.05$, comparison between SW620 cells and S5 cells. 


\section{CD31 and D2-40 expression levels exhibited increasing trends with the epithelial-mesenchymal transition potential of CRC cell lines}

We then investigated the link between CD31 and D2-40 expression and the metastatic potential in cell lines in vitro. Three CRC cell lines, namely SW480, SW620 and S5, were selected for this purpose, as they exhibit escalating potential of epithelial-mesenchymal transition (EMT). ${ }^{18,19}$ The expression of epithelial marker E-cadherin was gradually decreased from SW480, SW620 to S5 cells (Fig. 2A), while the mesenchymal marker N-cadherin exhibited the opposite trend of change in the three cell lines (Fig. 2B). Protein levels of these two EMT markers were consistent with their transcripts (Fig. 2C), which further confirmed that SW480, SW620, and S5 CRC cell lines displayed gradually increasing EMT potentials. Next, CD31 and D2-40 messenger RNA expression were examined in these three CRC cell lines, both of which were also gradually elevated (Fig. 3), indicated that they indeed exhibited increasing trends with the EMT potential of CRC cells in vitro.

\section{CD31 and D2-40 are essential for EMT of CRC cells}

Next, to investigate the role of CD31 and D2-40 on EMT potential of CRC cells, overexpression study was conducted in SW480 cells, which displayed lowest EMT potential among the three cell lines used. Although expression levels of CD31 and D2-40 were greatly increased in the experiments (Fig. 4A and B), overexpressing either CD31 or D2-40 alone had little effect on SW480 cells in terms of EMT potential (Supplementary Fig. 1). However, simultaneous overexpression of both CD31 and D2-40 induced significantly elevated EMT in SW480 cells, as evidenced in expression profiles of EMT markers E-cadherin and $\mathrm{N}$-cadherin (Fig. 4C and D), as well as in attachment and detachment assays (Fig. 4E and F).

On the other hand, KD assay using short hairpin RNA
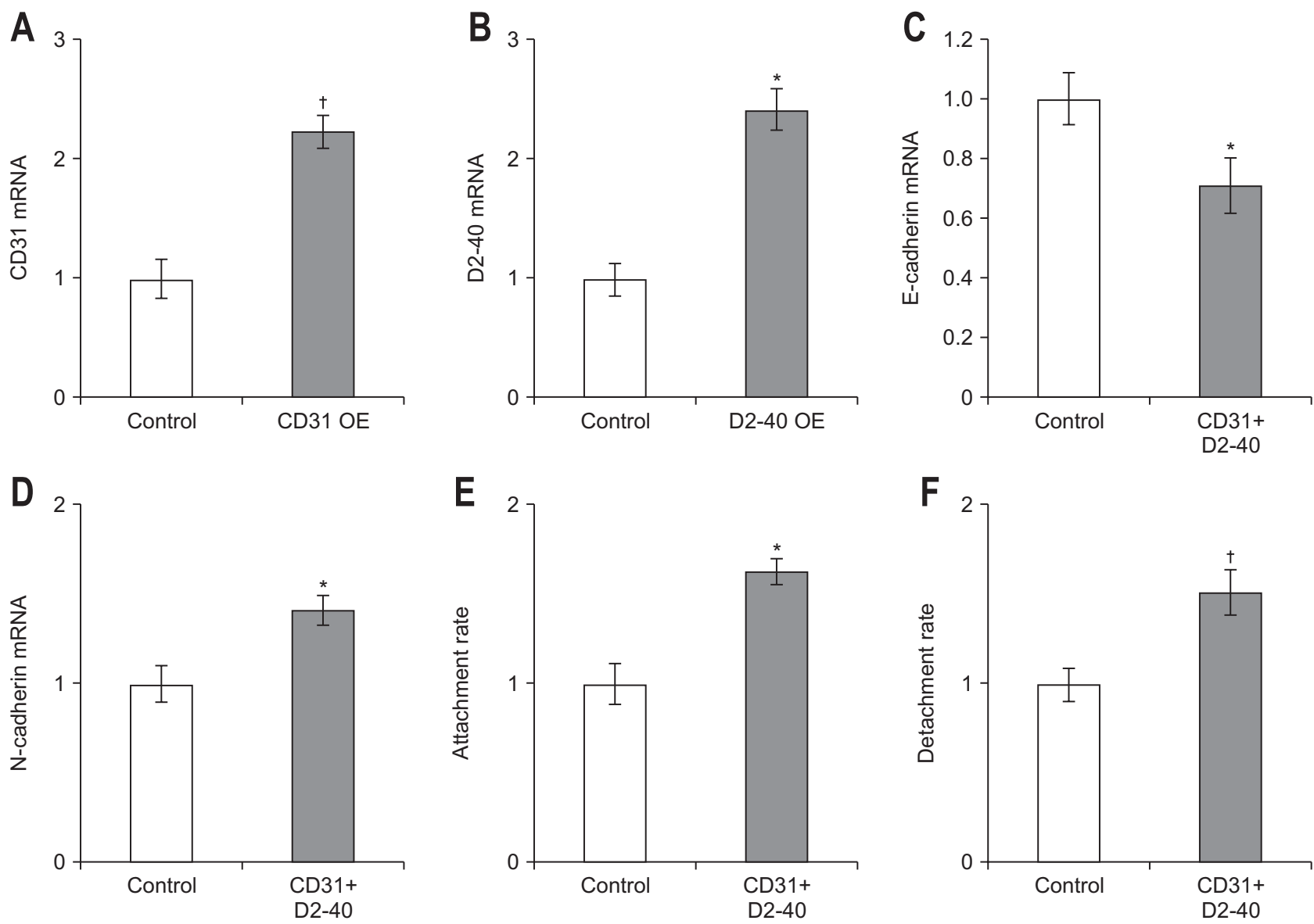

Fig. 4. CD31 and D2-40 overexpression (OE) induce epithelial-mesenchymal transition in SW480 colorectal cancer cells. (A) CD31 and (B) D2-40 messenger RNA (mRNA) expression levels were assessed by real-time polymerase chain reaction (RT-PCR) in SW480 cells transduced with either control or the corresponding OE lentivirus. (C) E-cadherin and (D) N-cadherin mRNA expression levels were assessed by RT-PCR in control SW480 cells and SW480 cells with simultaneous OE of CD31 and D2-40 (CD31+D2-40). (E) Cell attachment and (F) detachment rates were analyzed in control SW480 cells and SW480 cells with simultaneous OE of CD31 and D2-40 (CD31+D2-40). Values are the mean \pm SD of at least three biological replicates. ${ }^{*} p<0.05,{ }^{\dagger} p<0.01$, comparison of control cells with CD31 and/or D2-40 OE cells. 
against CD31 and D2-40 was performed in S5 cells (Fig. $5 \mathrm{~A}$ and $\mathrm{B}$ ), which showed highest EMT potential of all three cell lines. As expected, simultaneous silencing of CD31 and D2-40 resulted in severely compromised EMT phenotypes in S5 cells (Fig. 5C-F). Taken together, the above results demonstrated the essential role of both CD31 and D2-40 in the EMT of CRC cells in vitro.

\section{CD31 and D2-40 are essential for growth of xenograft tumor and peritoneal metastasis of CRC cells in vivo}

To further study the role of CD31 and D2-40 in tumorigenesis, a mouse model of xenograft CRC tumor were employed. First, SW480 cells with simultaneous overexpression of both CD31 and D2-40 were inoculated into the flanks of mice, and growth of the xenograft tumors were monitored for 15 days. As shown in Fig. 6A, CD31+D2-40 overexpression significant promoted growth rate of SW480 xenograft compared to the control. On day 15, weight of the tumors inoculated from SW480 cells overexpressing CD31+D2-40 was also heavier than control group (Fig. 6B). Second, S5 cells with double KD of CD31 and D2-40 $(\mathrm{CD} 31+\mathrm{D} 2-40 \mathrm{KD})$ were also inoculated into experimental mice, where the growth rates of these xenograft tumors were markedly lower than the control (Fig. 6C). Similarly, weight of the S5 CD31+D2-40 KD xenograft tumors on day 15 was also obviously reduced compared to control tumors (Fig. 6D).

The PCI has been employed by surgeons as a classic quantitative method to evaluate the extent of peritoneal cancer in the peritoneal cavity, and the PCI score tightly correlates with the patient's prognosis, ${ }^{20}$ and was also employed in this study to assess peritoneal metastasis in a mouse model of CRPM. ${ }^{16}$ Simply put, PCI of SW480 cells with $\mathrm{CD} 31+\mathrm{D} 2-40$ overexpression was significantly higher than the control (Fig. 7A), while that of S5 cells with
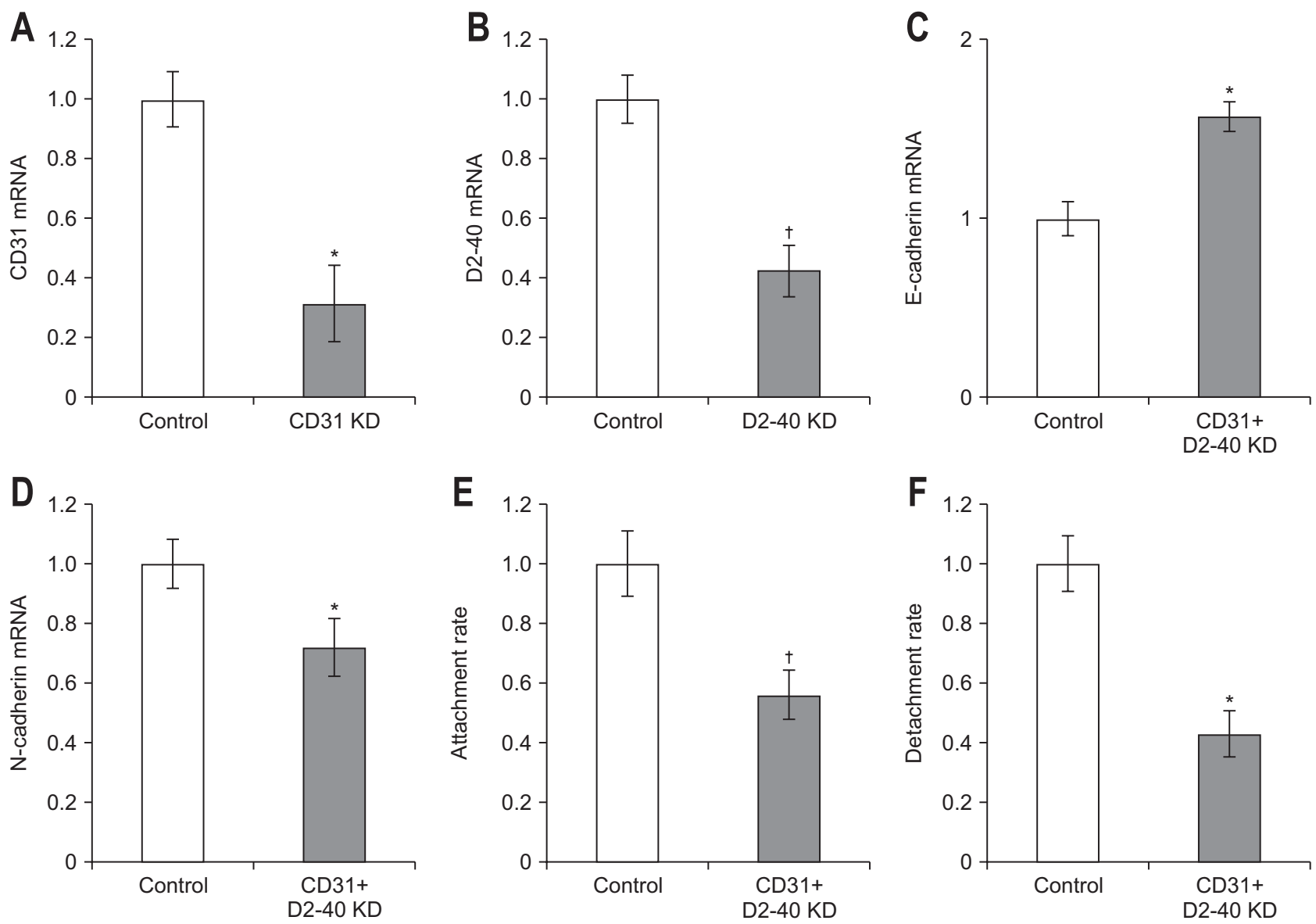

Fig. 5. CD31 and D2-40 knockdown (KD) inhibit epithelial-mesenchymal transition in S5 colorectal cancer cells. (A) CD31 and (B) D2-40 messenger RNA (mRNA) expression levels were assessed by real-time polymerase chain reaction (RT-PCR) in S5 cells transduced with either control or the corresponding short hairpin RNA lentivirus. (C) E-cadherin and (D) N-cadherin mRNA expression levels were assessed by RT-PCR in control S5 cells or S5 cells with simultaneous KD of CD31 and D2-40 (CD31+D2-40 KD). (E) Cell attachment and (F) detachment rates were analyzed in control S5 cells or S5 cells with simultaneous KD of CD31 and D2-40 (CD31+D2-40 KD). Values are the mean \pm SD of at least three biological replicates. ${ }^{*} p<0.05,{ }^{\dagger} p<0.01$, comparison of control cells with CD31 and/or D2-40 KD cells. 

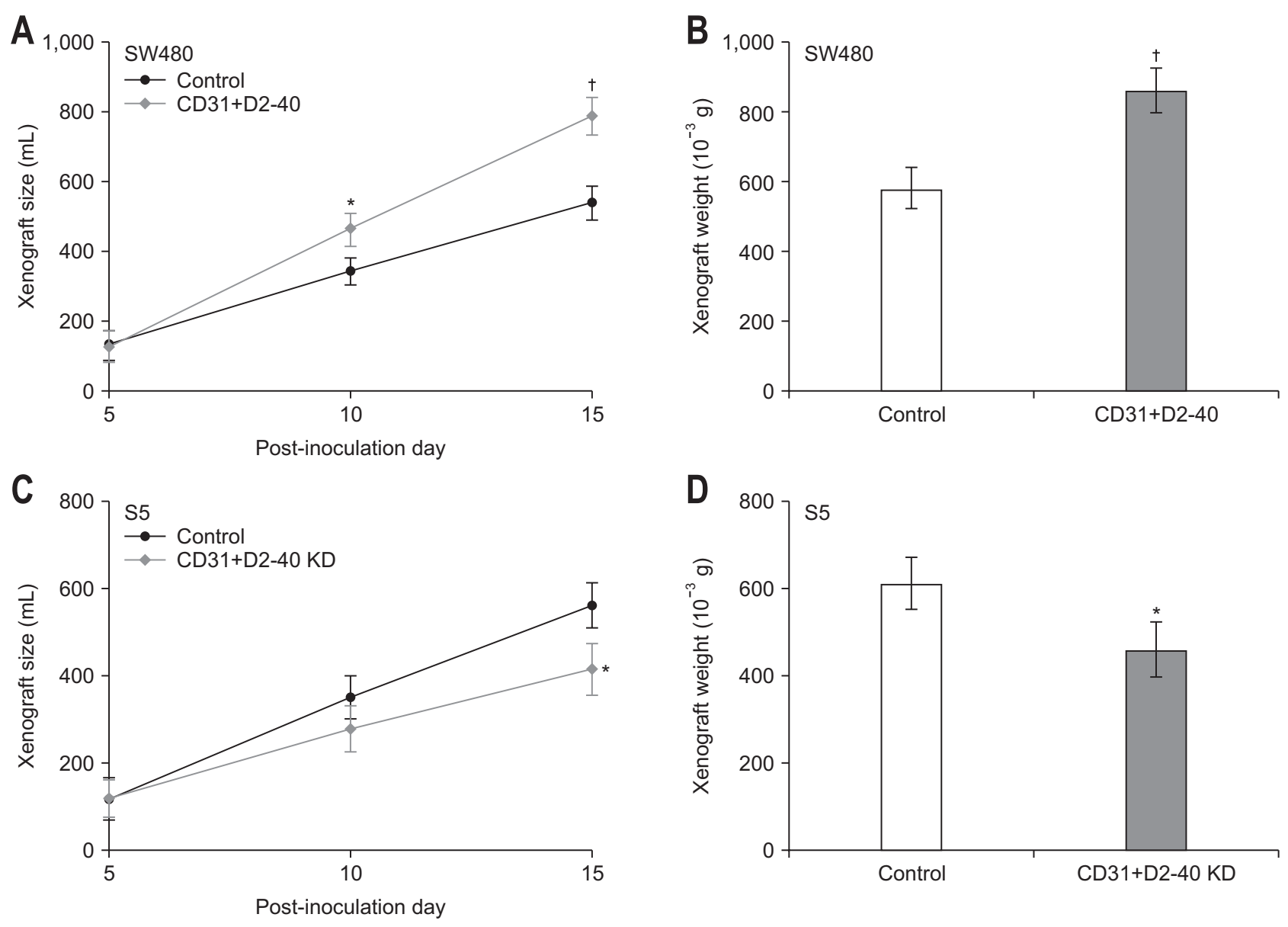

Fig. 6. CD31 and D2-40 are essential for the growth of xenograft tumors from inoculated colorectal cancer cells. (A) SW480 cells transduced with either control or CD31+D2-40 overexpression lentivirus were inoculated into mice ( $n=6$ per group), and the growth of xenograft tumors was monitored for up to 15 days. (B) On post inoculation day 15, mice were sacrificed, and the xenograft tumors were weighted. (C) S5 cells transduced with either control or CD31+D2-40 knockdown (CD31+D2-40 KD) lentivirus were inoculated into mice ( $n=6$ per group), and the growth of xenograft tumors was monitored for up to 15 days. (D) On post inoculation day 15 , mice were sacrificed, and the xenograft tumors were weighted. Values are the mean $\pm S D$ ( $n=6$ per group). ${ }^{*} p<0.05,{ }^{\dagger} p<0.01$, comparison between the two groups at the same time point.
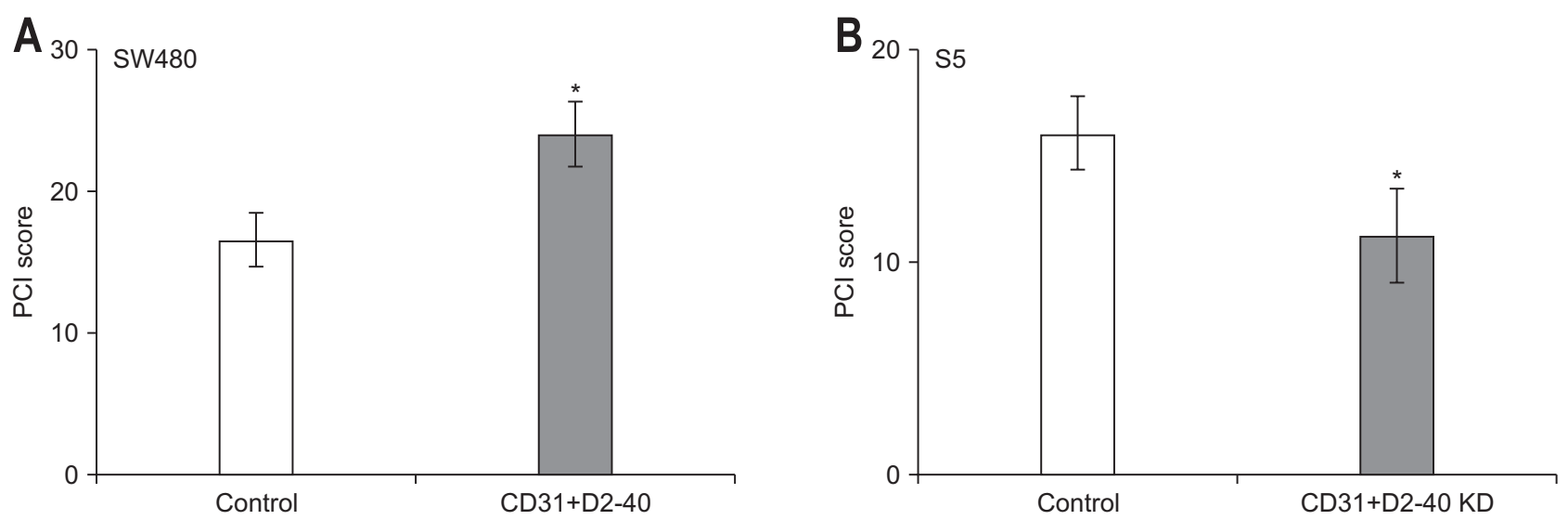

Fig. 7. CD31 and D2-40 are essential for peritoneal metastasis of colorectal cancer cells in vivo. (A) SW480 cells transduced with either control or CD31+D2-40 overexpression lentivirus were grafted into the peritoneal cavity of mice ( $\mathrm{n}=8$ per group). Fifteen days after engraftment, mice were sacrificed, and the peritoneal carcinomatosis index (PCI) score of the peritoneal cavity was measured. (B) S5 cells transduced with either control or CD31+D2-40 knockdown (CD31+D2-40 KD) lentivirus were grafted into the peritoneal cavity of mice ( $\mathrm{n}=8$ per group). Fifteen days after engraftment, mice were sacrificed, and the $\mathrm{PCl}$ score of the peritoneal cavity was measured. Values are the mean $\pm \mathrm{SD}$ ( $n=8$ per group). * $p<0.05$, comparison between the two groups. 
CD31+D2-40 KD was apparently lower than its respective control as well (Fig. 7B). The above data consistently supported the essential role of both CD31 and D2-40 in the in vivo growth, and more importantly the peritoneal metastasis of CRC cells.

\section{DISCUSSION}

Among various vascular markers, CD31, a 130-kDa transmembrane glycoprotein platelet endothelial cell adhesion molecule- 1 , is a member from the immunoglobulin superfamily. ${ }^{12}$ CD31 is expressed on platelets, monocytes, endothelial cells, and selected T-cell subsets, and more frequently found on blood vascular endothelial cells compared to lymphatic endothelial cells. ${ }^{21}$ D2-40, an IgG2a monoclonal antibody to the oncofetal M2A antigen, is often expressed in fetal testis and germ cell tumors. ${ }^{14}$ D2-40 is also expressed in lymphatic endothelium, and thus commonly used to selectively label lymphatic but not blood vessels. $^{22}$ D2-40 is now considered one of the most sensitive and specific markers for tumor cells in lymphatic vessels, which proves be a critical determinant of the outcome. ${ }^{23}$ These studies are certainly consistent with our observation in CRC patients, where not only expressions of CD31 and D2-40 were upregulated in tumor tissues of CRC patients, but in patients with CRPM their expressions were also noticeably higher. Echoing this elevated expression profile, CD31 and D2-40 also exhibited increasing trends with the EMT potential in three CRC cells examined in the current study. These data hinted the potential role of CD31 and D2-40 as prognosis markers especially in cases of CRPM.

In the field of CRC biology, D2-40 was first reported as a novel immunostaining marker for lymphatic vessels in colonic mucosa. ${ }^{24}$ Its specific involvement in CRC was only reported a decade later, where abnormal expression of D2-40 was observed in colorectal carcinomas, with certain links to clinicopathological characteristics and prognosis. ${ }^{25}$ On the other hand, CD31 was found to play a role in the prognosis of CRC cases, along with vascular endothelial growth factor and CD105. ${ }^{26}$ To the best of our knowledge, this is the first instance to implicate both CD31 and D2-40 in CRC.

We brought this discovery one step further, with the aim to reveal the molecular mechanism of CD31 and D240 involvement in CRPM. Tumor metastasis is constituted by selective, sequential, and interlinked steps, ${ }^{27}$ many of which feature conversions between two cellular states-the mesenchymal and epithelial phenotypes. The EMT, an essential regulatory developmental program, has been shown to exert pivotal and intricate functions to promote tumor invasion as well as metastasis in epithelium-derived carcinomas. ${ }^{28}$ Accumulating literature has demonstrated that the EMT plays complex and prominent roles in human CRC. ${ }^{28}$ Downregulation of E-cadherin is associated with poor tumor differentiation, lymph node metastasis, and a worse prognosis in patients with CRC, whereas upregulated expression of $\mathrm{N}$-cadherin strongly predicts lymph node metastasis and poor prognosis. ${ }^{29}$

To this end, three CRC cell lines with escalating EMT potential were employed. ${ }^{18,19}$ An increasing trend was observed between EMT potential, in terms of E-cadherin and $\mathrm{N}$-cadherin expression profiles, and levels of CD31 and D2-40 in these CRC cell lines. By overexpression and knockdown experiments, CD31 and D2-40 were found to be essential for EMT phenotypes in CRC cells, strongly implicating EMT as the downstream event of upregulated CD31 and D2-40 in CRC, at least in cells in vitro. Next, using the commonly employed xenograft tumor mouse model, we were able to demonstrate that both CD31 and D2-40 were also essential for CRC tumor growth in vivo. Importantly, to verify the roles of CD31 and D2-40 in CRPM, a recently reported mouse model of CRPM was utilized, in which CRC cells were directly grafted into the peritoneal cavity. ${ }^{16}$ As reported, the major quantitative endpoint to evaluate degree of CRPM, PCI was assessed in our experimental mice with the peritoneal cavity grafts. The results confirmed much severe extent of peritoneal metastasis with CD31 and D2-40 overexpression, which was otherwise attenuated by CD31+D2-40 KD. Altogether, our data indicated that CD31 and D2-40 were essential for growth of xenograft tumor and peritoneal metastasis in vivo.

In conclusion, CD31 and D2-40 contribute to CRPM by promoting EMT, which may serve as prognosis markers and therapeutic targets for treatment against CRC, particular among patients with peritoneal metastasis. Our current study has served as the first instance to implicate CD31 and D2-40 in CRPM, and additional investigations are currently underway to identify the effector signaling pathways downstream of CD31 and D2-40, as well as the mode of regulation and linkage between these two genes.

\section{CONFLICTS OF INTEREST}

No potential conflict of interest relevant to this article was reported. 


\section{ACKNOWLEDGEMENTS}

This work was supported by Jiangsu Provincial Health Commission (grant number: H2018055).

\section{AUTHOR CONTRIBUTIONS}

Study concept and design: P.N., X.J., H.H. Data acquisition: H.H., J.W., X.S., X.J. Drafting of the manuscript; critical revision of the manuscript for important intellectual content: J.L. Statistical analysis: X.Z., G.Z. Approval of final manuscript: all authors.

\section{ORCID}

Xinqiang Zhu https://orcid.org/0000-0002-7674-8555 Gang Zhou https://orcid.org/0000-0002-5175-4879 Peng Ni https://orcid.org/0000-0002-9397-8520 Xuetong Jiang https://orcid.org/0000-0002-3813-4675 Hailong Huang https://orcid.org/0000-0002-6201-8244 Jianqiang Wu https://orcid.org/0000-0002-6784-4692 Xiaohong Shi https://orcid.org/0000-0003-2726-9125 Xiaoling Jiang https://orcid.org/0000-0002-6635-590X Jianing Liu https://orcid.org/0000-0002-3373-4241

\section{REFERENCES}

1. Jemal A, Bray F, Center MM, Ferlay J, Ward E, Forman D. Global cancer statistics. CA Cancer J Clin 2011;61:69-90.

2. Brenner H, Kloor M, Pox CP. Colorectal cancer. Lancet 2014;383:1490-1502.

3. Issa IA, Noureddine M. Colorectal cancer screening: an updated review of the available options. World J Gastroenterol 2017;23:5086-5096.

4. Wang Y, Chen Z. Mutation detection and molecular targeted tumor therapies. STEMedicine 2020;1:e11.

5. Hubbard JM. Management of colorectal cancer in older adults. Clin Geriatr Med 2016;32:97-111.

6. Goéré D, Sourrouille I, Gelli M, Benhaim L, Faron M, Honoré C. Peritoneal metastases from colorectal cancer: treatment principles and perspectives. Surg Oncol Clin N Am 2018;27:563-583.

7. Segelman J, Granath F, Holm T, Machado M, Mahteme $\mathrm{H}$, Martling A. Incidence, prevalence and risk factors for peritoneal carcinomatosis from colorectal cancer. Br J Surg 2012;99:699-705.

8. Jayne DG, Fook S, Loi C, Seow-Choen F. Peritoneal carcinomatosis from colorectal cancer. Br J Surg 2002;89:1545-1550.
9. van Gestel YR, Thomassen I, Lemmens VE, et al. Metachronous peritoneal carcinomatosis after curative treatment of colorectal cancer. Eur J Surg Oncol 2014;40:963-969.

10. Goldhirsch A, Wood WC, Gelber RD, et al. Progress and promise: highlights of the international expert consensus on the primary therapy of early breast cancer 2007. Ann Oncol 2007;18:1133-1144.

11. Van den Eynden GG, van der Auwera I, van Laere SJ, et al. Distinguishing blood and lymph vessel invasion in breast cancer: a prospective immunohistochemical study. Br J Cancer 2006;94:1643-1649.

12. Mohammed RA, Ellis IO, Lee AH, Martin SG. Vascular invasion in breast cancer; an overview of recent prognostic developments and molecular pathophysiological mechanisms. Histopathology 2009;55:1-9.

13. Lee JA, Bae JW, Woo SU, Kim H, Kim CH. D2-40, podoplanin, and CD31 as a prognostic predictor in invasive ductal carcinomas of the breast. J Breast Cancer 2011;14:104111.

14. Breiteneder-Geleff S, Soleiman A, Kowalski H, et al. Angiosarcomas express mixed endothelial phenotypes of blood and lymphatic capillaries: podoplanin as a specific marker for lymphatic endothelium. Am J Pathol 1999;154:385-394.

15. Choi WW, Lewis MM, Lawson D, et al. Angiogenic and lymphangiogenic microvessel density in breast carcinoma: correlation with clinicopathologic parameters and VEGFfamily gene expression. Mod Pathol 2005;18:143-152.

16. Taibi A, Albouys J, Jacques J, et al. Comparison of implantation sites for the development of peritoneal metastasis in a colorectal cancer mouse model using non-invasive bioluminescence imaging. PLoS One 2019;14:e0220360.

17. Otto J, Jansen PL, Lucas S, Schumpelick V, Jansen M. Reduction of peritoneal carcinomatosis by intraperitoneal administration of phospholipids in rats. BMC Cancer 2007;7:104.

18. Mc Donnell S, Chaudhry V, Mansilla-Soto J, Zeng ZS, Shu WP, Guillem JG. Metastatic and non-metastatic colorectal cancer (CRC) cells induce host metalloproteinase production in vivo. Clin Exp Metastasis 1999;17:341-349.

19. Kuranami M, Cohen AM, Guillem JG. Analyses of protein kinase $\mathrm{C}$ isoform expression in a colorectal cancer liver metastasis model. Am J Surg 1995;169:57-64.

20. Maggiori L, Elias D. Curative treatment of colorectal peritoneal carcinomatosis: current status and future trends. Eur J Surg Oncol 2010;36:599-603.

21. Podgrabinska S, Braun P, Velasco P, Kloos B, Pepper MS, Skobe M. Molecular characterization of lymphatic endothelial cells. Proc Natl Acad Sci U S A 2002;99:16069-16074.

22. Fukunaga M. Expression of D2-40 in lymphatic endothelium of normal tissues and in vascular tumours. Histopathology 2005;46:396-402.

23. Kahn HJ, Marks A. A new monoclonal antibody, D2-40, for 
detection of lymphatic invasion in primary tumors. Lab Invest 2002;82:1255-1257.

24. Fogt F, Zimmerman RL, Ross HM, Daly T, Gausas RE. Identification of lymphatic vessels in malignant, adenomatous and normal colonic mucosa using the novel immunostain D2-40. Oncol Rep 2004;11:47-50.

25. Foda AA, El-Hawary AK, Hamed H. Aberrant expression of calretinin, D2-40 and mesothelin in mucinous and non-mucinous colorectal carcinomas and relation to clinicopathological features and prognosis. Pathol Oncol Res 2016;22:725-732.

26. Mohamed SY, Mohammed HL, Ibrahim HM, Mohamed EM, Salah M. Role of VEGF, CD105, and CD31 in the prognosis of colorectal cancer cases. J Gastrointest Cancer 2019;50:23-34.

27. Fidler IJ. The pathogenesis of cancer metastasis: the 'seed and soil' hypothesis revisited. Nat Rev Cancer 2003;3:453458.

28. Zhu W, Cai MY, Tong ZT, et al. Overexpression of EIF5A2 promotes colorectal carcinoma cell aggressiveness by upregulating MTA1 through C-myc to induce epithelialmesenchymaltransition. Gut 2012;61:562-575.

29. He X, Chen Z, Jia M, Zhao X. Downregulated E-cadherin expression indicates worse prognosis in Asian patients with colorectal cancer: evidence from meta-analysis. PLoS One 2013;8:e70858. 